\title{
Authority Control
}

compiled by Anna Appleman

Bargioni, Stefano. "From Authority Enrichment to AuthorityBox. Applying RDA in a Koha Environment.” JLIS.it 11, no. 1 (2020): 175-89. dx.doi.org/10.4403/jlis.it-12595.

Bitter, Colin and Yuji Tosaka. "Genre/Form Access in Library Catalogs: A Survey on the Current State of LCGFT Usage.” Library Resources \& Technical Services 64, no. 2 (2020): 44.

Cohen, Ahava. "Luck Is What Happens When Preparation Meets Opportunity: Building Israel's Multilingual, Multiscript Authority Database.” Cataloging \& Classification Quarterly 58, no. 7 (October 2020): 632-50.

Danskin, Alan. "The Anglo-American Authority File: A PCC Story.” Cataloging \& Classification Quarterly 58, nos. 3/4 (April 2020): 221-29.

Wolf, Stacey. “Automating Authority Control Processes.” Code4Lib Journal 47 (February 17, 2020). 